\title{
Synthesis, Characterization, Anti-Bacterial and Anti-Fungal Activities of New Quinoxaline 1,4-di-N-Oxide Derivatives
}

\author{
Dalia Hussein Soliman \\ Pharmaceutical Chemistry Department, Faculty of Pharmacy, Al-Azhar University, Cairo, Egypt \\ Email: odihss@yahoo.com
}

Received September 4, 2013; revised October 8, 2013; accepted October 24, 2013

Copyright (C) 2013 Dalia Hussein Soliman. This is an open access article distributed under the Creative Commons Attribution License, which permits unrestricted use, distribution, and reproduction in any medium, provided the original work is properly cited.

\begin{abstract}
A new series of quinoxaline 1,4-di-N-oxides were synthesized and evaluated for their antibacterial and antifungal activities. The best result was demonstrated by 3-amino-N-(4-methoxyphenyl)-2-quinoxalinecarboxamide 1,4-di-N-oxide 4e, MIC $(0.24 \mu \mathrm{g} / \mathrm{ml})$ against Aspergillus fumigatus, and $(0.12 \mu \mathrm{g} / \mathrm{ml})$ against Streptococcus pneumonia.
\end{abstract}

Keywords: Quinoxaline 1,4-di-N-Oxide; Beirut Reaction; Benzofuroxanes; $\beta$-Ketoamide; $\beta$-Cyanoamide; Antibacterial; Antifungal

\section{Introduction}

There is a growing need to develop new antibacterial agents in order to overcome the emergence of bacterial resistance to antibiotic therapy. Quinoxaline 1,4-di-Noxide is a nucleus that displays a wide range of activities. Quinoxaline 1,4-di- $N$-oxides and their derivatives demonstrated excellent activities as antibacterial [1-5], antiplasmodial [2,6,7], antifungal [8] and as antimycobacterial [9-13]. Encouraged by these reported activities and with the aim of searching for new, broad spectrum and more potent antimicrobial compounds which can improve the current chemotherapeutic treatments, 20 new quinoxaline 1,4-di-Noxide derivatives were synthesized and evaluated. In most of the quinoxaline 1,4-di-N-oxides reported as antibacterial and/or antimycobacterial, the carboxamide in position 2 was a common feature $[11,13]$. Therefore, the newly synthesized compounds 3a-n possessed a carboxamide in position 2 that was substituted with various aryl moieties bearing both electron-withrawing and electron donating groups. This series of quinoxaline carboxamide was prepared from unsubstituted benzofuroxane, 3a-g to be compared to their 7-chloro counterparts $3 \mathbf{h}-\mathbf{n}$ in order to study the effect of substitution of chlorine atom in position 7 .

\section{Results and Discussion}

\subsection{Chemistry}

The synthetic route for the preparation of the quinoxaline
1,4-di-N-oxide were obtained by the Beirut reaction[14, 15]. This reaction, which has been referred to as the Beirut reaction, is an excellent method for preparing these heterocyclic compounds. Benzofuroxanes 1a,b were obtained by previously described methods $[13,16]$. Thus, the appropriate BFO reacted with the corresponding $\beta$-ketoamide (2a,b) or $\beta$-cyanoamide (3a-n) in the presence anhydrous potassium carbonate as a catalyst (Scheme 1). The use of inorganic catalyst for this reaction provided better means of purification with considerably good yields, whereas the reaction of BFO with cyanoacetamide was better carried out using triethylamine as a catalyst and dimethylfomamide as the solvent. These two intermediates, $\beta$-ketoamide and $\beta$-cyanoamide, were prepared by condensation of either ethylacetoacetate or ethylcyanoacetae with different amines [11,12,17]. When the target compounds were prepared from monosubstituted-BFO, the formation of isomeric quinoxaline 1,4-di-N-oxides was observed. In most cases, the 7-substituted isomer prevailed over 6-substituted isomer, and when the methoxy substituted quinoxalines were prepared, only the 7-isomer was obtained, as previously described $[18,19]$.

Moreover, in order to investigate the antimicrobial activity of fused tricyclic and tetracyclic di-N-oxides derived from quinoxaline 1,4-di-N-oxide the phenazine derivative 5 and the indenoquinoxaline 6 were synthesized. The Beirut reaction was similarly followed using 


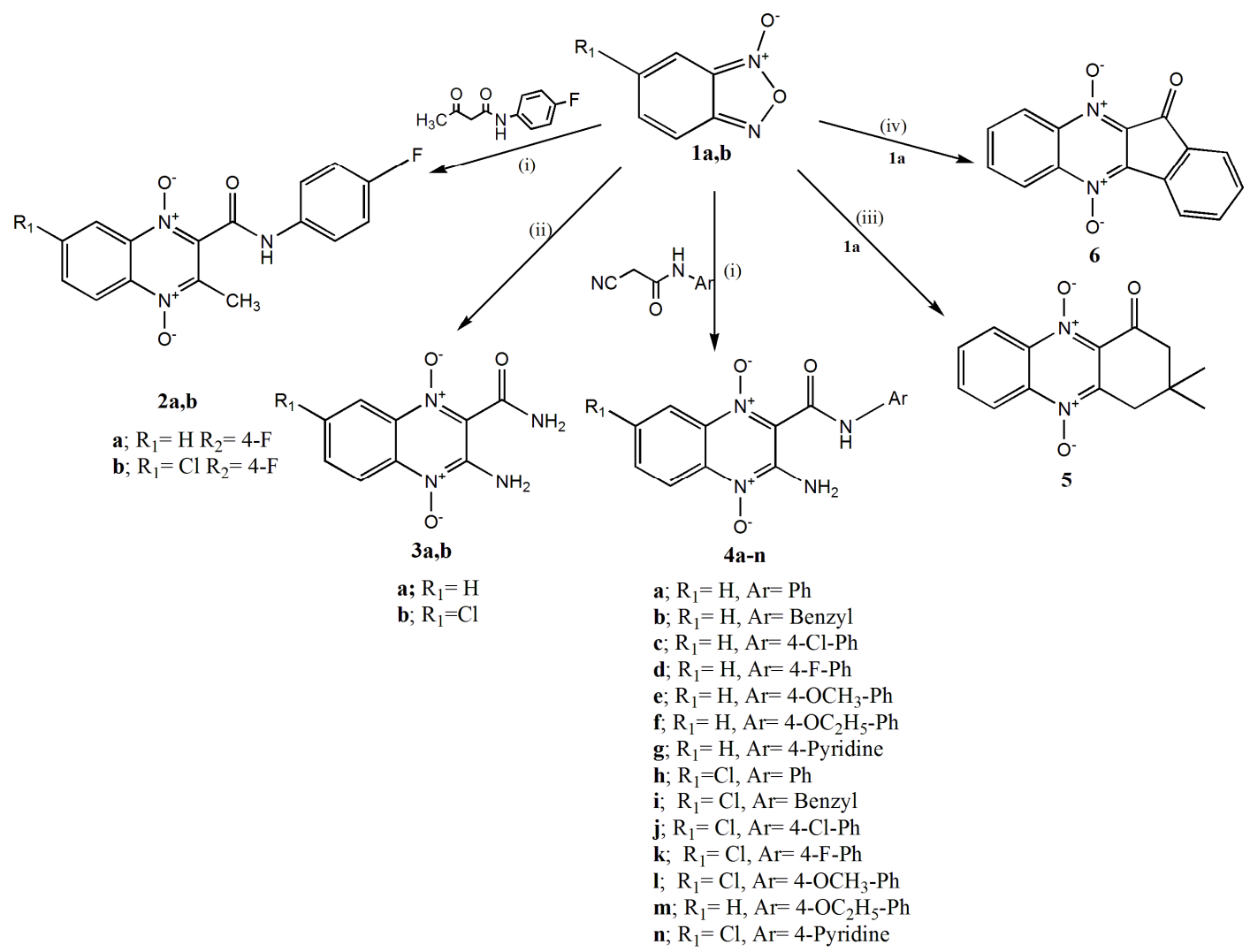

Scheme 1. Synthesis of target compounds. Conditions: (i): $\mathrm{EtOH}, \mathrm{K}_{2} \mathrm{CO}_{3}$; (ii): $\mathrm{CNCH}_{2} \mathrm{CONH}_{2}$, DMF/TEA $0^{\circ} \mathrm{C}$; (iii) dimidone, ammonia gas, EtOH, $40^{\circ} \mathrm{C}, 12 \mathrm{hr}$; (iv) indanedione, morpholine, EtOH, stirring, $12 \mathrm{hr}$.

cyclic diketones such as indandione or dimidone. All the prepared compounds were characterized in light of their microanalysis and spectral data including IR, ${ }^{1} \mathrm{H}$ NMR and mass spectrum.

\subsection{Microbiology}

The newly synthesized compounds were tested for their antibacterial and antifungal activities. These assays were performed at the Regional Center for Mycology and Biotechnology, Antimicrobial unit test organisms, Cairo, Egypt. Compounds $\mathbf{4 i}$ and $\mathbf{4 k}$ were previously synthesized [17] but they were prepared again in order to perform the microbiological testing on them and compare them to their unsubstituted counterparts. The tested compounds were evaluated for their antifungal activity against (Aspergillus fumigatus (RCMB 02568), Syncephalastrum racemosum (RCMB 05922), Geotricum candidum ( $R C M B$ 05097) Candida albicans (RCMB 05036)). The antibacterial activity was evaluated against (Gram positive: Streptococcus pneumonia (RCMB 010010), Bacillis subtilis (RCMB 010067)) and Gram negative (Pseudomonas aeruginosa (RCMB 010046), Escherichia coli (RCMB 010052)). Amphotericin B was taken as a reference for the antifungal effect, while ampicillin was the standard used for the evaluation of anti- bacterial activity against gram positive bacteria and gentamicin was used as a standard in assessing the activity of the tested compounds against gram negative bacteria. The results expressed as the mean zone of inhibition in $\mathrm{mm} \pm$ standard deviation beyond well diameter $(6 \mathrm{~mm})$ produced on the microorganisms using $(10 \mathrm{mg} / \mathrm{ml})$ concentration of tested samples, shown in Table 1.

The initial screening of the tested compounds showed promising activity of some of the compounds which encouraged the determination of their minimum inhibitory concentration (MIC) (Table 2). The best results were demonstrated by compound $\mathbf{4 e}$ both as antifungal and against gram positive bacteria, it possessed double the activity of the standard, amphotericin B against Aspergillus fumigatus, $(0.24$ and $0.49 \mu \mathrm{g} / \mathrm{ml}$, respectively)and ampicillin against Streptococcus pneumonia $(0.12$ and $0.24 \mu \mathrm{g} / \mathrm{ml}$, respectively). This compound has also displayed 4 times the activity of amphotericin B against Syncephalastrum racemosum, $(7.81,1.95 \mu \mathrm{g} / \mathrm{ml}$, respectively). Other derivatives also possessed remarkable activity against Syncephalastrum racemosum as $\mathbf{4 i}$ which displayed double the inhibitory effect of the standard (3.9, $7.81 \mu \mathrm{g} / \mathrm{ml}$, respectively), while $\mathbf{2 a}$ and $\mathbf{6}$ were as active as the amphotericin B. Moderate activity against gram positive bacteria Streptococcus pneumonia was also demonstrated by compounds $\mathbf{3 b}$ and $\mathbf{6}$. However, this 
Table 1. Antifungal and antibacterial activity of the newly synthesized compounds.

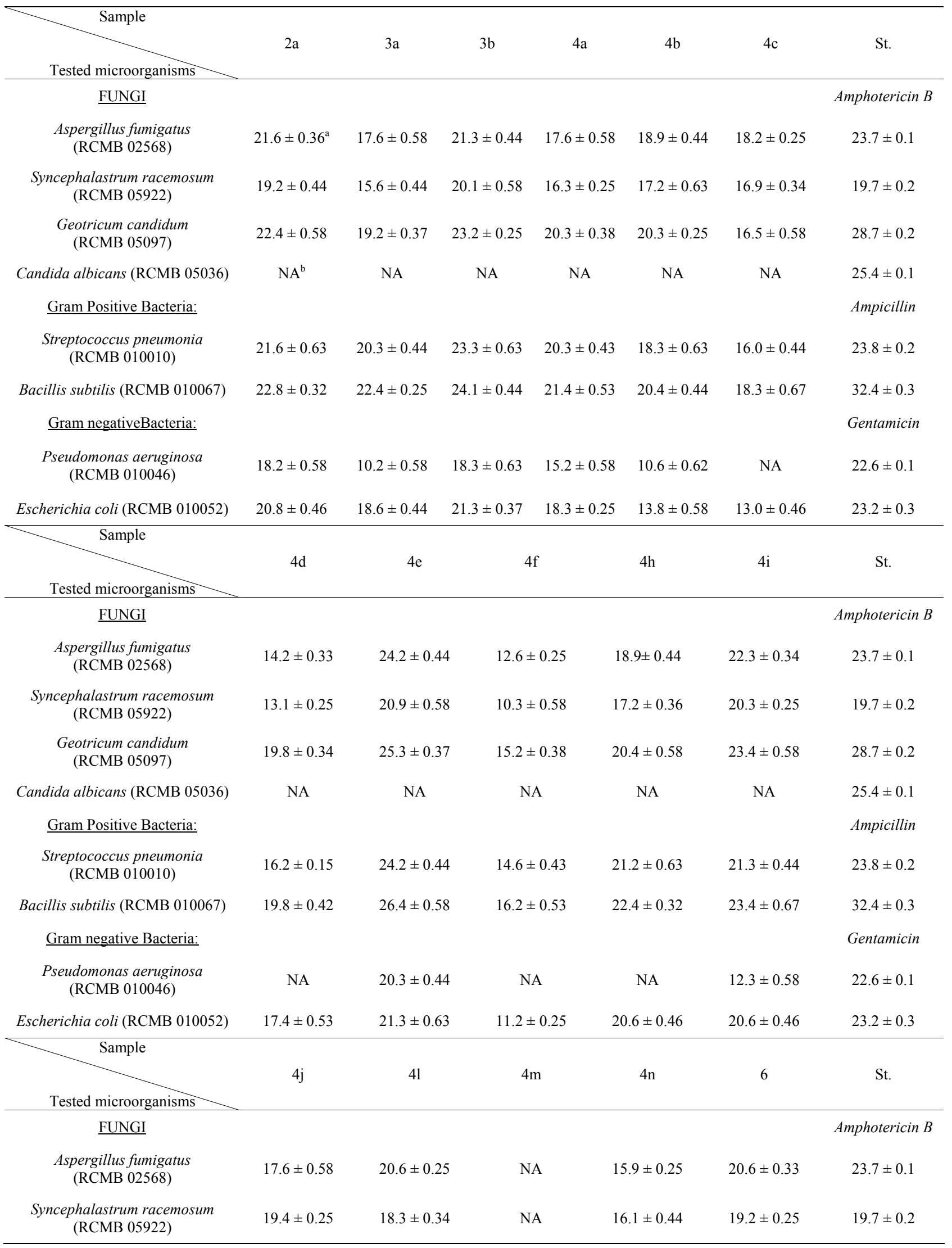


Continued

\begin{tabular}{|c|c|c|c|c|c|c|}
\hline $\begin{array}{l}\text { Geotricum candidum } \\
\quad(\text { RCMB 05097) }\end{array}$ & $19.9 \pm 0.38$ & $21.6 \pm 0.58$ & NA & $19.2 \pm 0.63$ & $22.4 \pm 0.34$ & $28.7 \pm 0.2$ \\
\hline Candida albicans (RCMB 05036) & NA & NA & NA & NA & NA & $25.4 \pm 0.1$ \\
\hline Gram Positive Bacteria: & & & & & & Ampicillin \\
\hline $\begin{array}{l}\text { Streptococcus pneumonia } \\
\text { (RCMB 010010) }\end{array}$ & $17.7 \pm 0.43$ & $21.2 \pm 0.44$ & $12.3 \pm 0.44$ & $19.6 \pm 0.25$ & $22.4 \pm 0.15$ & $23.8 \pm 0.2$ \\
\hline Bacillis subtilis (RCMB 010067) & $19.3 \pm 0.53$ & $22.6 \pm 0.67$ & $14.6 \pm 0.63$ & $20.3 \pm 0.63$ & $23.2 \pm 0.42$ & $32.4 \pm 0.3$ \\
\hline Gram negativeBacteria: & & & & & & Gentamicin \\
\hline $\begin{array}{l}\text { Pseudomonas aeruginosa } \\
\quad \text { (RCMB 010046) }\end{array}$ & NA & NA & NA & NA & $16.8 \pm 0.37$ & $22.6 \pm 0.1$ \\
\hline Escherichia coli (RCMB 010052) & $13.7 \pm 0.25$ & $19.9 \pm 0.46$ & $14.4 \pm 0.25$ & $16.9 \pm 0.44$ & $20.4 \pm 0.53$ & $23.2 \pm 0.3$ \\
\hline
\end{tabular}

${ }^{a}$ Data are expressed in the form of mean zone of inhibition in $\mathrm{mm} \pm$ standard deviation beyond well diameter (6 mm) produced on a range of environmental and clinically pathogenic microorganisms using $(10 \mathrm{mg} / \mathrm{ml})$ concentration of tested samples; ${ }^{\mathrm{b}} \mathrm{NA}$ : No activity.

Table 2. Antimicrobial and antifungal activity as MICS $(\mu \mathrm{g} / \mathrm{ml})$ of tested compounds against tested microorganisms.

\begin{tabular}{|c|c|c|c|c|c|c|c|c|c|c|c|}
\hline \multirow{2}{*}{ Tested microorganisms } & \multicolumn{10}{|c|}{$\operatorname{MIC}(\mu \mathrm{g} / \mathrm{ml})^{\mathrm{a}}$} & \multirow{2}{*}{ St. } \\
\hline & $2 \mathrm{a}$ & $3 a$ & $3 b$ & $4 a$ & $4 b$ & $4 \mathrm{e}$ & $4 \mathrm{~h}$ & $4 \mathrm{i}$ & 41 & 6 & \\
\hline$\underline{\text { FUNGI }}$ & & & & & & & & & & & Amphotericin B \\
\hline $\begin{array}{l}\text { Aspergillus fumigatus } \\
\text { (RCMB 02568) }\end{array}$ & 0.98 & 31.25 & 1.95 & 31.25 & 31.25 & 0.24 & 7.81 & 0.98 & 1.95 & 3.9 & 0.49 \\
\hline $\begin{array}{c}\text { Syncephalastrum racemosum } \\
\text { (RCMB 05922) }\end{array}$ & 7.81 & 62.5 & 3.9 & 62.5 & 62.5 & 1.95 & 31.25 & 3.9 & 15.63 & 7.81 & 7.81 \\
\hline $\begin{array}{l}\text { Geotricum candidum } \\
\quad \text { (RCMB 05097) }\end{array}$ & 0.98 & 7.81 & 0.49 & 3.9 & 3.9 & 0.12 & 3.9 & 0.49 & 1.95 & 0.98 & 0.03 \\
\hline Candida albicans (RCMB 05036) & NA & NA & NA & NA & NA & NA & NA & NA & NA & NA & 0.12 \\
\hline Gram Positive Bacteria: & & & & & & & & & & & Ampicillin \\
\hline $\begin{array}{l}\text { Streptococcus pneumonia } \\
\text { (RCMB 010010) }\end{array}$ & 1.95 & 3.9 & 0.49 & 3.9 & 15.63 & 0.12 & 1.95 & 1.95 & 1.95 & 0.98 & 0.24 \\
\hline Bacillis subtilis (RCMB 010067) & 0.98 & 0.98 & 0.24 & 1.95 & 3.9 & 0.06 & 0.98 & 0.49 & 0.98 & 0.49 & 0.007 \\
\hline Gram negativeBacteria: & & & & & & & & & & & Gentamicin \\
\hline $\begin{array}{l}\text { Pseudomonas aeruginosa } \\
\text { (RCMB 010046) }\end{array}$ & 15.63 & 500 & 15.63 & 125 & 500 & 3.9 & NA & 125 & NA & 31.25 & 0.98 \\
\hline Escherichia coli (RCMB 010052) & 3.9 & 15.63 & 1.95 & 15.63 & 125 & 1.95 & 3.9 & 3.9 & 7.81 & 3.9 & 0.98 \\
\hline
\end{tabular}

${ }^{\mathrm{a}}$ Minimum inhibitory concentration $(\mu \mathrm{g} / \mathrm{ml})$; ${ }^{\mathrm{b}} \mathrm{NA}$ : No activity.

series of compounds had no effect on Candida albicans and were also ineffective against gram negative bacteria. Moreover, compounds $\mathbf{2 b}, \mathbf{4 g}$, $\mathbf{4 k}$ and $\mathbf{5}$ showed no activity against most of the tested strains.

A simple SAR study reveals certain features that could improve the activity such as the amino group in position 3 , quinoxaline unsubstituted in position 6 , the electrondonating substituents on the phenyl amino displayed better effect than electron withdrawing. As for the fused derivatives the tetracyclic indenoquinoxaline 6 was better than the tricyclic phenazine 5 .

\section{Conclusion}

In this study, certain novel quinoxaline 1,4-di-N-oxide derivatives were synthesized and evaluated for their antbacterial and antifungal activities. The 3-amino-N-(4methoxyphenyl)-2-quinoxalinecarboxamide $1,4-\mathrm{di}-\mathrm{N}-$ oxide 4e demonstrated the best result with MIC 0.12 $\mu \mathrm{g} / \mathrm{ml}$ compared to ampicillin $(0.24 \mu \mathrm{g} / \mathrm{ml})$ against Streptococcus pneumonia and MIC $0.24 \mu \mathrm{g} / \mathrm{ml}$ compared to Amphotericin B $(0.49 \mu \mathrm{g} / \mathrm{ml})$ against Aspergillus fumigatus. However, there series of compounds were inactive 
against gram negative bacteria as well as candida albicans

\section{Experimental}

\subsection{Chemistry}

\subsubsection{Chemical Methods}

All the solvents used were commercially available and distilled before use. Reactions were monitored by thinlayer chromatography (TLC) on silica gel plates (60 F254), visualizing with ultraviolet. Infra red spectra (KBr) were recorded on FT-IR 5300 spectrophotometer and Perkin Elmer spectrum RXIFT-IR system $\left(v, \mathrm{~cm}^{-1}\right)$. ${ }^{1}$ HNMR spectra were recorded on Varian Gemini spectrophotometer $(300 \mathrm{MHz})$ in $\mathrm{DMSO}-\mathrm{d}_{6}$ or $\mathrm{CDCl}_{3}$ as solvent. Proton chemical shifts (d) are relative to tetramethylsilane (TMS, d =0.00) as internal standard and expressed in ppm. Spin multiplicities are given as $\mathrm{s}$ (singlet), $\mathrm{d}$ (doublet), $\mathrm{t}$ (triplet), and $\mathrm{m}$ (multiplet) as well as $b$ (broad). Coupling constants $(\mathrm{J})$ are given in hertz. Melting points were determined by using melting point apparatus and are uncorrected. MS spectra were obtained on a GC Ms-QP 1000 EX mass spectrometer at $70 \mathrm{eV}$. Microanalyses were performed using a $\mathrm{C} \mathrm{H} \mathrm{N} \mathrm{S/O} \mathrm{ana-}$ lyzer. Elemental data are within $\pm 0.4 \%$ of the theoretical values. All yields reported areunoptimized. The chemical reagents used in synthesis were purchased from Fluka, Sigma and Aldrich.

\subsubsection{General Procedure for Preparation of Compounds 2a,b}

A warm solution of 5(6)-benzofuroxane $\mathbf{1 a , b}$ and the appropriate acetoacetanilide $(0.01 \mathrm{~mol})$ in ethanol $(50$ $\mathrm{mL}$ ) was stirred at room temperature in the presence of catalytic amount of potassium carbonate. The yellow products precipitated over a period of $2-4 \mathrm{~h}$. The residual product was triturated with water, extracted with ethylacetate then the organic solvent was dried over anhydrous sodium sulphate and removed in vacuo to give yellow crystals that were recrystallized out from ethanol.

1) 2-N-(4-Fluorophenyl)-3-methyl-2-quinoxaline carboxamide 1,4-di-N-oxide $2 \mathbf{a}$.

Yield (78\%); mp $221^{\circ} \mathrm{C}$. IR (KBr, $\left.\mathrm{cm}^{-1}\right)$ : $1651(\mathrm{C}=\mathrm{O})$, $1575(\mathrm{C}=\mathrm{N}), 3290(\mathrm{NH}), 1215,1295(\mathrm{NO}) .{ }^{1} \mathrm{H}$ NMR spectrum (DMSO-d6), $\delta$, ppm $2.63\left(3 \mathrm{H}, \mathrm{s}, \mathrm{CH}_{3}\right), 11.04$ (1H, s, NH-D $2 \mathrm{O}$ exchangable), $7.23(2 \mathrm{H}, \mathrm{d}, \mathrm{Ar}-\underline{\mathrm{H}}, \mathrm{J}=8.7$ $\mathrm{Hz}), 7.6(2 \mathrm{H}, \mathrm{d}, \mathrm{Ar}-\underline{\mathrm{H}}, \mathrm{J}=8.7 \mathrm{~Hz}), 7.99\left(2 \mathrm{H}, \mathrm{d}, \mathrm{H}_{5}, \mathrm{H}_{8}\right)$, $8.52\left(\mathrm{~m}, 2 \mathrm{H}, \mathrm{H}_{6}, \mathrm{H}_{7}\right) . \mathrm{MS}(\mathrm{m} / \mathrm{z}): 313\left(\mathrm{M}^{+}\right), 281\left(\mathrm{M}^{+}-\mathrm{O}_{2}\right)$, $202\left(\mathrm{M}^{+}-4-\mathrm{F}-\mathrm{Ph}-\mathrm{NH}\right), 171\left(\mathrm{M}^{+}-\mathrm{O}_{2},-4-\mathrm{F}-\mathrm{Ph}-\mathrm{NH}\right), 129$ (quinoxaline $^{+}$). Found, \%: C; H; N. C 61.47; H 3.66; N, 13.12. $\mathrm{C}_{16} \mathrm{H}_{12} \mathrm{FN}_{3} \mathrm{O}_{3}$. Calc., \%: C, 61.34; H, 3.86; N, 13.41 .

2) 7-chloro-2-N-(4-fluorophenyl)-3-methyl-2-quinoxaline carboxamide 1,4-dioxide $\mathbf{2} \mathbf{b}$.

Yield (85\%); mp $243^{\circ} \mathrm{C}$. IR (KBr, $\left.\mathrm{cm}^{-1}\right)$ : $1670(\mathrm{C}=\mathrm{O})$,
$1561(\mathrm{C}=\mathrm{N}), 3211(\mathrm{NH}), 1334(\mathrm{NO}) . \mathrm{MS}(\mathrm{m} / \mathrm{z}): 349$ $\left(\mathrm{M}^{+}\right), 205\left(\mathrm{M}^{+}-\mathrm{NH}-\mathrm{C}_{6} \mathrm{H}_{5}-4-\mathrm{F},-\mathrm{O}_{2}\right)$. Found, \%: C; H; N. C 54.47; H 3.66; N, 13.01. $\mathrm{C}_{16} \mathrm{H}_{13} \mathrm{ClFN}_{3} \mathrm{O}_{3}$. Calc., \%: C, $54.95 ; \mathrm{H}, 3.75 ; \mathrm{N}, 12.01$.

\subsubsection{General Procedure for Preparation of Compounds 3a,b}

A mixture of 5(6)-substituted benzofuroxane 1a,b and cyanoacetamide $(0.01 \mathrm{~mol})$ was stirred for $10 \mathrm{~min}$ at $0^{\circ} \mathrm{C}$. Over the cooled suspension, a solution of triethylamine (5 drops) in dimethylformamide was added. The mixture was allowed to stand at room temperature over $24 \mathrm{~h}$ and then filtered off. The red solid product was filtered and recrystallized from ethanol.

1) 3-Amino-2-quinoxalinecarboxamide 1,4-dioxide 3a.

Yield $71 \%$; mp $260^{\circ} \mathrm{C}-265^{\circ} \mathrm{C}$. IR $\left(\mathrm{KBr}, \mathrm{cm}^{-1}\right): 1660$ $(\mathrm{C}=\mathrm{O}), 1640(\mathrm{C}=\mathrm{N}), 3388,3224\left(2 \mathrm{NH}_{2}\right), 1254(\mathrm{NO}) .{ }^{1} \mathrm{H}$ NMR spectrum (DMSO-d6), $\delta$, ppm $5.6\left(\mathrm{~s}, 2 \mathrm{H}, \mathrm{NH}_{2}\right.$ ), $8.53\left(\mathrm{~s}, 2 \mathrm{H}, \mathrm{CO}-\mathrm{NH}_{2}\right), 7.26-8.2$ (m, 4H, quinox-H). Found, \%: C; H; N. C 49.47; H 2.96; N, 25.40. $\mathrm{C}_{9} \mathrm{H}_{8} \mathrm{~N}_{4} \mathrm{O}_{3} \mathrm{C}, 49.09 ; \mathrm{H}, 3.66 ; \mathrm{N}, 25.45$.

2) 3-Amino-7-chloro-2-quinoxaline carboxamide 1,4dioxide $\mathbf{3 b}$.

Yield $92 \%$; mp. $270^{\circ} \mathrm{C}-271^{\circ} \mathrm{C}$. IR $\left(\mathrm{KBr}, \mathrm{cm}^{-1}\right): 1715$ $(\mathrm{C}=\mathrm{O}), 1640(\mathrm{C}=\mathrm{N}), 1319,1340(\mathrm{NO}), 3356,3454,3512$ $\left(2 \mathrm{NH}_{2}\right) .{ }^{1} \mathrm{H}$ NMR spectrum (DMSO-d6), $\delta$, ppm $5.76(\mathrm{~s}$,

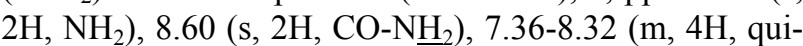
nox-H). Found, \%: C; H; N. C 42.47; H 2.66; N, 22.12. $\mathrm{C}_{9} \mathrm{H}_{7} \mathrm{ClN}_{4} \mathrm{O}_{3} \mathrm{C}, 42.45 ; \mathrm{H}, 2.77 ; \mathrm{N}, 22.00$.

\subsubsection{General Procedure for Preparation of Compounds 4a-n}

A warm solution of 5(6)-benzofuroxane 1a,b and the appropriate cyanoacetanilide $(0.01 \mathrm{~mol})$ in ethanol $(50$ $\mathrm{mL}$ ) was stirred at room temperature in the presence of catalytic amount of potassium carbonate. The solutions turned red and then they were stirred at room temperature for 1 - $5 \mathrm{hr}$ depending on the BFO and the cyanoacetanilides. The residue obtained was triturated with waterand then extracted with dichloromethane. The organic phase was dried with anhydrous sodium sulphate and filtered. The solvent was removed in vacuo and the red-brown precipitate was recrystallized out from ethanol.

1) 3-Amino-N-phenyl-2-quinoxaline carboxamide 1,4di-N-oxide 4a.

Yield $(67 \%)$; mp $180^{\circ} \mathrm{C}$. IR $\left(\mathrm{KBr}, \mathrm{cm}^{-1}\right): 1668(\mathrm{C}=\mathrm{O})$, $1563(\mathrm{C}=\mathrm{N}), 3210(\mathrm{NH}), 3256,3390\left(\mathrm{NH}_{2}\right), 1301(\mathrm{NO})$. ${ }^{1} \mathrm{H}$ NMR spectrum (DMSO-d6), $\delta$, ppm $4.2\left(\mathrm{~s}, 2 \mathrm{H}, \mathrm{NH}_{2}\right)$, $7.1\left(\mathrm{t}, 1 \mathrm{H}, \mathrm{H}_{4}{ }^{\prime}\right), 7.26\left(\mathrm{t}, 2 \mathrm{H}, \mathrm{H}_{3}{ }^{\prime}+\mathrm{H}_{5}{ }^{\prime}\right), 7.49\left(\mathrm{~d}, 2 \mathrm{H}, \mathrm{H}_{2}\right.$ ' $\left.+\mathrm{H}_{6}{ }^{\prime}\right), 7.85-7.97\left(\mathrm{~m}, 2 \mathrm{H}, \mathrm{H}_{6}+\mathrm{H}_{7}\right), 8.1\left(\mathrm{~d}, 2 \mathrm{H}, \mathrm{H}_{5}+\mathrm{H}_{8}\right)$, 10.79 (s, $1 \mathrm{H}, \mathrm{NH}-\mathrm{D}_{2} \mathrm{O}$ exchangeable) ppm. Found, \%: C; H; N. C 60.47; H 4.66; N, 18.12. $\mathrm{C}_{15} \mathrm{H}_{12} \mathrm{~N}_{4} \mathrm{O}_{3}$. Calc., \%: C, 60.81; H, 4.08; N, 18.91 .

2) 3-Amino-N-benzyl-2-quinoxaline carboxamide 1,4- 


\section{di-N-oxide $\mathbf{4 b}$}

Yield (62\%); mp $192^{\circ} \mathrm{C}$. IR $\left(\mathrm{KBr}, \mathrm{cm}^{-1}\right): 1659(\mathrm{C}=\mathrm{O})$, $1547(\mathrm{C}=\mathrm{N}), 3230-33313\left(\mathrm{NH}, \mathrm{NH}_{2}\right), 1298(\mathrm{NO}) .{ }^{1} \mathrm{H}$ NMR spectrum (DMSO-d6), $\delta$, ppm 4.32, $4.64(2 \mathrm{~d}, 2 \mathrm{H}$, $\left.\mathrm{N}-\underline{\mathrm{H}}_{2}\right), 7.26-7.46(\mathrm{~m}, 5 \mathrm{H}$, phenyl- $\underline{\mathrm{H}}), 7.52-7.55$ (m, $\left.2 \mathrm{H}, \mathrm{H}_{6}+\mathrm{H}_{7}\right), 8.48$ (s, $2 \mathrm{H}, \mathrm{NH}_{2}-\mathrm{D}_{2} \mathrm{O}$ exchangeable), 8.61 (t, $1 \mathrm{H}, \mathrm{NH}-\mathrm{D}_{2} \mathrm{O}$ exchangeable) ppm. Found, \%: $\mathrm{C} ; \mathrm{H} ; \mathrm{N}$. C 61.47; H 4.66; N, 18.12. $\mathrm{C}_{16} \mathrm{H}_{14} \mathrm{~N}_{4} \mathrm{O}_{3}$. Calc., \%: C, 61.93; H, 4.55; N, 18.06 .

3) 3-Amino- $N$-(4-chlorophenyl)-2-quinoxaline carboxamide $1,4-d i-N$-oxide $\mathbf{4 c}$.

Yield (83\%); mp 209 ${ }^{\circ} \mathrm{C}$. IR ( $\left.\mathrm{KBr}, \mathrm{cm}^{-1}\right): 1673(\mathrm{C}=\mathrm{O})$, $1631(\mathrm{C}=\mathrm{N}), 3269(\mathrm{NH}), 3347,3401\left(\mathrm{NH}_{2}\right), 1245(\mathrm{NO})$. ${ }^{1} \mathrm{H}$ NMR spectrum (DMSO-d6), $\delta$, ppm $7.05\left(\mathrm{t}, \mathrm{H}_{6}+\mathrm{H}_{7}\right)$, $7.2\left(\mathrm{~d}, 2 \mathrm{H}, \mathrm{H}_{5}+\mathrm{H}_{8}\right), 7.74\left(\mathrm{~d}, 2 \mathrm{H}, \mathrm{H}_{3}{ }^{\prime}+\mathrm{H}_{5}{ }^{\prime}\right), 7.79(\mathrm{~d}, 2 \mathrm{H}$, $\mathrm{H}_{2}{ }^{\prime}+\mathrm{H}_{4}{ }^{\prime}$ ), 10.12 (s, $1 \mathrm{H}, \mathrm{NH}-\mathrm{D}_{2} \mathrm{O}$ exchangeable), 8.0 (s, $2 \mathrm{H}, \mathrm{NH}_{2}-\mathrm{D}_{2} \mathrm{O}$ exchangeable) ppm. Found, \%: C; H; N. C 54.37; H 3.66; N, 16.12. $\mathrm{C}_{15} \mathrm{H}_{11} \mathrm{ClN}_{4} \mathrm{O}_{3}$. Calc., \%: $\mathrm{C}$, 54.47; H, 3.35; N, 16.94 .

4) 3-Amino- $N$-(4-fluorophenyl)-2-quinoxaline carboxamide $1,4-d i-N$-oxide $\mathbf{4 d}$.

Yield $(81 \%) ; \mathrm{mp} 210^{\circ} \mathrm{C}$. IR $\left(\mathrm{KBr}, \mathrm{cm}^{-1}\right): 1670(\mathrm{C}=\mathrm{O})$, $1621(\mathrm{C}=\mathrm{N}), 3256(\mathrm{NH}), 3327,3398\left(\mathrm{NH}_{2}\right), 1305(\mathrm{NO})$. ${ }^{1} \mathrm{H}$ NMR spectrum (DMSO-d6), $\delta$, ppm $7.08\left(\mathrm{t}, 2 \mathrm{H}, \mathrm{H}_{6}+\right.$ $\left.\mathrm{H}_{7}\right), 7.62\left(\mathrm{~d}, 2 \mathrm{H}, \mathrm{H}_{5}+\mathrm{H}_{8}\right), 7.74\left(\mathrm{~d}, 2 \mathrm{H}, \mathrm{H}_{3}{ }^{\prime}+\mathrm{H}_{5}{ }^{\prime}, 7.99\right.$ (d, $\left.2 \mathrm{H}, \mathrm{H}_{2}{ }^{\prime}+\mathrm{H}_{4}{ }^{\prime}\right), 10.10$ (s, 1H, NH- $\mathrm{D}_{2} \mathrm{O}$ exchangeable), 10.85 (br. s, $2 \mathrm{H}, \mathrm{NH}_{2}-\mathrm{D}_{2} \mathrm{O}$ exchangeable) ppm. $\mathrm{MS}(\mathrm{m} / \mathrm{z}$ ): $314\left(\mathrm{M}^{+}\right), 282\left(\mathrm{M}^{+}-\mathrm{O}_{2}\right), 172\left(\mathrm{M}^{+}-\mathrm{O}_{2}-\mathrm{NH}-\mathrm{C}_{6} \mathrm{H}_{5}-4-\mathrm{F},\right)$. Found, \%: C; H; N. C 57.47; H 3.66; N, 17.12 . $\mathrm{C}_{15} \mathrm{H}_{11} \mathrm{FN}_{4} \mathrm{O}_{3}$. Calc., \%: C, 57.33; H, 3.53; N, 17.83 .

5) 3-Amino- $N$-(4-methoxyphenyl)-2-quinoxaline carboxamide 1,4-di-N-oxide $\mathbf{4 e .}$

Yield (76\%); mp $195^{\circ} \mathrm{C}$. IR $\left(\mathrm{KBr}, \mathrm{cm}^{-1}\right): 1662(\mathrm{C}=\mathrm{O})$, $1611(\mathrm{C}=\mathrm{N}), 3250-3378\left(\mathrm{NH}, \mathrm{NH}_{2}\right), 1325(\mathrm{NO}) .{ }^{1} \mathrm{H}$ NMR spectrum (DMSO-d6), $\delta$, ppm 3.77(s, 3H, $\mathrm{OC}_{3}$ ), 3.89 (br. s, $2 \mathrm{H}, \mathrm{NH}_{2}-\mathrm{D}_{2} \mathrm{O}$ exchangeable), $6.92-7.278$ (m, $8 \mathrm{H}, \mathrm{Ar}-\mathrm{H}+$ quinox-H), 78.06(d, $\left.2 \mathrm{H}, \mathrm{H}_{2}{ }^{\prime}+\mathrm{H}_{4}{ }^{\prime}\right), 10.32(\mathrm{~s}$, $1 \mathrm{H}, \mathrm{NH}-\mathrm{D}_{2} \mathrm{O}$ exchangeable), ppm. Found, \%: C; H; N. C 58.47; H 4.66; N, 17.12. $\mathrm{C}_{16} \mathrm{H}_{14} \mathrm{~N}_{4} \mathrm{O}_{4}$. Calc., \%: C, 58.89; $\mathrm{H}, 4.32 ; \mathrm{N}, 17.17$.

6) 3-Amino- $N$-(4-ethoxyphenyl)-2-quinoxaline carboxamide $1,4-d i-\mathrm{N}$-oxide $\mathbf{4 f}$.

Yield (79\%); mp $198^{\circ} \mathrm{C}$. IR $\left(\mathrm{KBr}, \mathrm{cm}^{-1}\right)$ : $1660(\mathrm{C}=\mathrm{O})$, $1598(\mathrm{C}=\mathrm{N}), 3259-3421\left(\mathrm{NH}, \mathrm{NH}_{2}\right), 1355(\mathrm{NO}) .{ }^{1} \mathrm{H}$ NMR spectrum (DMSO-d6), $\delta$, ppm 1.29 (t, 3H, $\left.\mathrm{OCH}_{2} \mathrm{CH}_{3}\right), 4.02$ (q, $\left.2 \mathrm{H}, \mathrm{OCH}_{2} \mathrm{CH}_{3}\right), 6.97\left(\mathrm{~m}, 2 \mathrm{H}, \mathrm{H}_{6}+\right.$ $\left.\mathrm{H}_{7}\right), 7.28\left(\mathrm{~d}, 2 \mathrm{H}, \mathrm{H}_{3}{ }^{\prime}+\mathrm{H}_{5}{ }^{\prime}\right), 7.73\left(\mathrm{~d}, 2 \mathrm{H}, \mathrm{H}_{5}+\mathrm{H}_{8}\right), 8.04$ (d, $\left.2 \mathrm{H}, \mathrm{H}_{2}{ }^{\prime}+\mathrm{H}_{4}{ }^{\prime}\right), 10.67$ (s, $1 \mathrm{H}, \mathrm{NH}-\mathrm{D}_{2} \mathrm{O}$ exchangeable), 12.12 (br. s, $2 \mathrm{H}, \mathrm{NH}_{2}-\mathrm{D}_{2} \mathrm{O}$ exchangeable), ppm. Found, \%: C; H; N. C 59.47; H 4.66; N, 16.12. $\mathrm{C}_{17} \mathrm{H}_{16} \mathrm{~N}_{4} \mathrm{O}_{4}$. Calc., \%: C, 59.99; H, 4.74; N, 16.46.

7) 3-Amino- $N$-(pyridine-4-yl)-2-quinoxaline carboxamide 1,4-di- $\mathrm{N}$-oxide $\mathbf{4 g}$.

Yield (59\%); mp $211^{\circ} \mathrm{C}-214^{\circ} \mathrm{C}$. IR $\left(\mathrm{KBr}, \mathrm{cm}^{-1}\right): 1663$
$(\mathrm{C}=\mathrm{O}), 1604(\mathrm{C}=\mathrm{N}), 3278-3435\left(\mathrm{NH}, \mathrm{NH}_{2}\right), 1345(\mathrm{NO})$. ${ }^{1} \mathrm{H}$ NMR spectrum (DMSO-d6), $\delta$, ppm $7.41\left(\mathrm{~d}, 2 \mathrm{H}, \mathrm{H}_{5}+\right.$ $\left.\mathrm{H}_{8}\right), 7.71\left(\mathrm{~m}, 2 \mathrm{H}, \mathrm{H}_{6}+\mathrm{H}_{7}\right), 8.22\left(\mathrm{~d}, 2 \mathrm{H}, \mathrm{H}_{3}{ }^{\prime}+\mathrm{H}_{5}{ }^{\prime}\right), 8.37$ (d, $\left.2 \mathrm{H}, \mathrm{H}_{2}{ }^{\prime}+\mathrm{H}_{4}{ }^{\prime}\right), 10.18$ (s, $1 \mathrm{H}, \mathrm{NH}-\mathrm{D}_{2} \mathrm{O}$ exchangeable), 12.46 (br. s, $2 \mathrm{H}, \mathrm{NH}_{2}-\mathrm{D}_{2} \mathrm{O}$ exchangeable), ppm. Found, \%: C; H; N. C 56.47; H 3.66; N, 23.12. $\mathrm{C}_{14} \mathrm{H}_{11} \mathrm{~N}_{5} \mathrm{O}_{3}$. Calc., \%: C, 56.56; H, 3.73; N, 23.56.

8) 3-Amino-7-Chloro-N-phenyl-2-quinoxaline carboxamide 1,4-di- $\mathrm{N}$-oxide $\mathbf{4 h}$.

Yield (83\%); $\mathrm{mp} 223^{\circ} \mathrm{C}-224^{\circ} \mathrm{C}$. IR $\left(\mathrm{KBr}, \mathrm{cm}^{-1}\right): 1670$ $(\mathrm{C}=\mathrm{O}), 1615(\mathrm{C}=\mathrm{N}), 3221(\mathrm{NH}), 3266,3421\left(\mathrm{NH}_{2}\right), 1353$ (NO). ${ }^{1} \mathrm{H}$ NMR spectrum (DMSO-d6), $\delta$, ppm 7.43 (m, $\left.1 \mathrm{H}, \mathrm{H}_{4}{ }^{\prime}\right), 7.66\left(\mathrm{~m}, 2 \mathrm{H}, \mathrm{H}_{3}{ }^{\prime}+\mathrm{H}_{5}{ }^{\prime}\right), 7.89\left(\mathrm{~d}, 2 \mathrm{H}, \mathrm{H}_{2}{ }^{\prime}+\mathrm{H}_{6}{ }^{\prime}\right)$, $7.92\left(\mathrm{~d}, 1 \mathrm{H}, \mathrm{H}_{5}\right), 8.02\left(\mathrm{~s}, 1 \mathrm{H}, \mathrm{H}_{8}\right), 8.29\left(\mathrm{~d}, 1 \mathrm{H}, \mathrm{H}_{6}\right), 10.70$ (s, $1 \mathrm{H}, \mathrm{NH}-\mathrm{D}_{2} \mathrm{O}$ exchangeable), $13.6\left(\mathrm{~s}, 2 \mathrm{H}, \mathrm{NH}_{2}-\mathrm{D}_{2} \mathrm{O}\right.$ exchangeable), ppm. Found, \%: C; H; N. C 54.47; H 3.66; $\mathrm{N}, 16.12 . \mathrm{C}_{15} \mathrm{H}_{11} \mathrm{ClN}_{4} \mathrm{O}_{3} \mathrm{C}, 54.47 ; \mathrm{H}, 3.35 ; \mathrm{N}, 16.94$.

9) 3-Amino-7-Chloro-N-benzyl-2-quinoxaline carboxamide 1,4-di-N-oxide $\mathbf{4 i}$.

Yield (84\%); mp $214^{\circ} \mathrm{C}-216^{\circ} \mathrm{C}$. IR $\left(\mathrm{KBr}, \mathrm{cm}^{-1}\right): 1664$ $(\mathrm{C}=\mathrm{O}), 1572(\mathrm{C}=\mathrm{N}), 3223-3345\left(\mathrm{NH}, \mathrm{NH}_{2}\right), 1331(\mathrm{NO})$. ${ }^{1} \mathrm{H}$ NMR spectrum (DMSO-d6), $\delta$, ppm $4.63(\mathrm{~d}, 2 \mathrm{H}$, $\left.\mathrm{N}-\underline{\mathrm{H}}_{2}\right), 7.26-7.46,7.79\left(2 \mathrm{~m}, 7 \mathrm{H}\right.$, phenyl- $\left.\underline{\mathrm{H}}, \mathrm{H}_{5}+\mathrm{H}_{6}\right)$, $7.99\left(\mathrm{~s}, 1 \mathrm{H}, \mathrm{H}_{8}\right), 8.48\left(\mathrm{~s}, 2 \mathrm{H}, \mathrm{NH}_{2}-\mathrm{D}_{2} \mathrm{O}\right.$ exchangeable), 8.81 (t, $1 \mathrm{H}, \mathrm{NH}-\mathrm{D}_{2} \mathrm{O}$ exchangeable) ppm. Found, \%: $\mathrm{C}$, 55.34; H, 3.70; N, 16.12. $\mathrm{C}_{16} \mathrm{H}_{13} \mathrm{ClN}_{4} \mathrm{O}_{3}$. Calc., \%: C, 55.74; H, 3.80; N, 16.25 .

10) 3-Amino-7-Chloro- $N$-(4-chlorophenyl)-2-quinoxaline carboxamide $1,4-d i-N$-oxide $\mathbf{4 j}$.

Yield (89\%); mp 240 ${ }^{\circ}$. IR ( $\left.\mathrm{KBr}, \mathrm{cm}^{-1}\right): 1674(\mathrm{C}=\mathrm{O})$, $1587(\mathrm{C}=\mathrm{N}), 3224(\mathrm{NH}), 3276,3470\left(\mathrm{NH}_{2}\right), 1335(\mathrm{NO})$. ${ }^{1} \mathrm{H}$ NMR spectrum (DMSO-d6), $\delta$, ppm $7.46\left(\mathrm{~d}, 1 \mathrm{H}, \mathrm{H}_{2}\right.$ ' $\left.+\mathrm{H}_{6}{ }^{\prime}\right), 7.59\left(\mathrm{~d}, 1 \mathrm{H}, \mathrm{H}_{6}\right), 7.73\left(\mathrm{~d}, 2 \mathrm{H} \mathrm{H}_{3}{ }^{\prime}+\mathrm{H}_{5}{ }^{\prime}\right), 7.83$ (s, $\left.1 \mathrm{H}, \mathrm{H}_{8}\right), 7.88\left(\mathrm{~d}, 1 \mathrm{H}, \mathrm{H}_{5}\right), 10.89\left(\mathrm{~s}, 1 \mathrm{H}, \mathrm{NH}-\mathrm{D}_{2} \mathrm{O}\right.$ exchangeable), 13.69(s, 2H, $\mathrm{NH}_{2}-\mathrm{D}_{2} \mathrm{O}$ exchangeable), ppm. MS (m/z): $364\left(\mathrm{M}^{+}\right), 332\left(\mathrm{M}^{+}-\mathrm{O}_{2}\right), 191\left(\mathrm{M}^{+}-\mathrm{O}_{2}-\mathrm{NH}-\right.$ $\left.\mathrm{C}_{6} \mathrm{H}_{5}-4-\mathrm{Cl}-\mathrm{NH}\right)$. Found, \%: C 49.47; H 2.66; N, 15.12 . $\mathrm{C}_{15} \mathrm{H}_{10} \mathrm{Cl}_{2} \mathrm{~N}_{4} \mathrm{O}_{3} \mathrm{C}$, 49.34; H, 2.76; N, 15.34 .

11) 3-Amino-7-Chloro- $N$-(4-flourorophenyl)-2-quinoxaline carboxamide 1,4-di-N-oxide $\mathbf{4 k}$.

Yield (91\%); mp $244^{\circ} \mathrm{C}$. IR $\left(\mathrm{KBr}, \mathrm{cm}^{-1}\right): 1669(\mathrm{C}=\mathrm{O})$, $1602(\mathrm{C}=\mathrm{N}), 3210-3380\left(\mathrm{NH}, \mathrm{NH}_{2}\right), 1301(\mathrm{NO}) . \mathrm{MS}$ $(\mathrm{m} / \mathrm{z}): 348\left(\mathrm{M}^{+}\right), 350(\mathrm{M}+2), 332\left(\mathrm{M}^{+}-\mathrm{O}_{2}\right), 221\left(\mathrm{M}^{+}-\mathrm{O}_{2-}\right.$ NH- $\left.\mathrm{C}_{6} \mathrm{H}_{5}-4-\mathrm{F}\right)$. Found, \%: C; H; N. C 51.47; H 2.66; N, 16.12. $\mathrm{C}_{15} \mathrm{H}_{10} \mathrm{ClFN}_{4} \mathrm{O}_{3} \mathrm{C}, 51.66 ; \mathrm{H}, 2.89 ; \mathrm{N}, 16.07$.

12) 3-Amino-7-Chloro- $N$-(4-methoxyphenyl)-2-quinoxalinecarboxamide 1,4-di-N-oxide $\mathbf{4 l}$.

Yield (82\%); mp 219 ${ }^{\circ} \mathrm{C}$. IR $\left(\mathrm{KBr}, \mathrm{cm}^{-1}\right)$ : $1664(\mathrm{C}=\mathrm{O})$, $1520(\mathrm{C}=\mathrm{N}), 3298(\mathrm{NH}), 3620,3589\left(\mathrm{NH}_{2}\right), 1248(\mathrm{NO})$. ${ }^{1} \mathrm{H}$ NMR spectrum (DMSO-d6), $\delta$, ppm $3.75(\mathrm{~s}, 3 \mathrm{H}$, $\left.\mathrm{OCH}_{3}\right), 6.97\left(\mathrm{~d}, 2 \mathrm{H}, \mathrm{H}_{3}{ }^{\prime}+\mathrm{H}_{5}{ }^{\prime}\right), 7.62\left(\mathrm{~d}, 2 \mathrm{H}, \mathrm{H}_{2}{ }^{\prime}+\mathrm{H}_{6}{ }^{\prime}\right)$, 7.81 (s. $\left.1 \mathrm{H}, \mathrm{H}_{8}\right), 8.00\left(\mathrm{~d}, 1 \mathrm{H}, \mathrm{H}_{5}\right), 8.22\left(\mathrm{D}, 1 \mathrm{H}, \mathrm{H}_{6}\right)$, 10.27 (s, 1H, NH- ${ }_{2} \mathrm{O}$ exchangeable), 13.60 (s, $2 \mathrm{H}, \mathrm{NH}_{2}-$ $\mathrm{D}_{2} \mathrm{O}$ exchangeable), ppm. MS (m/z): $360\left(\mathrm{M}^{+}\right), 362(\mathrm{M}+$ 
2), $206\left(\mathrm{M}^{+}-\mathrm{O}_{2}-\mathrm{NH}-\mathrm{C}_{6} \mathrm{H}_{5}-4-\mathrm{OCH}_{3}\right)$. Found, \%: C; $\mathrm{H} ; \mathrm{N}$. C 53.47; $\mathrm{H}$ 3.66; N, 15.12. $\mathrm{C}_{16} \mathrm{H}_{13} \mathrm{ClN}_{4} \mathrm{O}_{4} \mathrm{C}, 53.27 ; \mathrm{H}$, $3.63 ; \mathrm{N}, 15.53$.

13) 3-Amino-7-Chloro-N-(4-ethoxyphenyl)-2-quinoxaline carboxamide 1,4-di-N-oxide $4 \mathrm{~m}$.

Yield (84\%); $\mathrm{mp} 219^{\circ} \mathrm{C}-220^{\circ} \mathrm{C}$. IR ( $\left.\mathrm{KBr}, \mathrm{cm}^{-1}\right): 1663$ $(\mathrm{C}=\mathrm{O}), 1613(\mathrm{C}=\mathrm{N}), 3255,3389\left(\mathrm{NH}, \mathrm{NH}_{2}\right), 1351(\mathrm{NO})$. ${ }^{1} \mathrm{H}$ NMR spectrum (DMSO-d6), $\delta$, ppm 3.75 (s, 3H, $\left.\mathrm{OCH}_{3}\right), 6.97\left(\mathrm{~d}, 2 \mathrm{H}, \mathrm{H}_{3}{ }^{\prime}+\mathrm{H}_{5}{ }^{\prime}\right), 7.62\left(\mathrm{~d}, 2 \mathrm{H}, \mathrm{H}_{2}{ }^{\prime}+\mathrm{H}_{6}{ }^{\prime}\right)$, 7.81 (s. $\left.1 \mathrm{H}, \mathrm{H}_{8}\right), 8.00$ (d, $\left.1 \mathrm{H}, \mathrm{H}_{5}\right), 8.22\left(\mathrm{D}, 1 \mathrm{H}, \mathrm{H}_{6}\right)$, 10.27 (s, 1H, NH-D ${ }_{2} \mathrm{O}$ exchangeable), 13.60 (s, 2H, $\mathrm{NH}_{2}-$ $\mathrm{D}_{2} \mathrm{O}$ exchangeable), ppm. MS (m/z): $360\left(\mathrm{M}^{+}\right), 362(\mathrm{M}+$ 2), $206\left(\mathrm{M}^{+}-\mathrm{O}_{2}-\mathrm{NH}-\mathrm{C}_{6} \mathrm{H}_{5}-4-\mathrm{OCH}_{3}\right)$. Found, \%: C; $\mathrm{H}$; N. C 53.07; H 3.26; N, 15.12. $\mathrm{C}_{16} \mathrm{H}_{13} \mathrm{ClN}_{4} \mathrm{O}_{4} \mathrm{C}, 53.27 ; \mathrm{H}$, $3.63 ; \mathrm{N}, 15.53$.

14) 3-Amino-7-Chloro-N-(pyridin-4-yl)-2-quinoxaline carboxamide 1,4-di- $N$-oxide $4 \mathbf{n}$.

Yield (63\%); mp $223^{\circ} \mathrm{C}$. IR $\left(\mathrm{KBr}, \mathrm{cm}^{-1}\right)$ : $1658(\mathrm{C}=\mathrm{O})$, $1554(\mathrm{C}=\mathrm{N}), 3202-3325\left(\mathrm{NH}, \mathrm{NH}_{2}\right), 1251(\mathrm{NO}) .{ }^{1} \mathrm{H}$ NMR spectrum (DMSO-d6), $\delta$, ppm 7.03 (d, 1H, $\mathrm{H}_{5}$ ), 7.91 (d, $\left.1 \mathrm{H}, \mathrm{H}_{6}\right), 8.14$ (d, 2H, $\mathrm{H}_{2}{ }^{\prime}+\mathrm{H}_{6}$ '), 8.17 (s. $1 \mathrm{H}, \mathrm{H}_{8}$ ), $8.34\left(\mathrm{~d}, 2 \mathrm{H}, \mathrm{H}_{3}{ }^{\prime}+\mathrm{H}_{5}{ }^{\prime}\right), 10.27$ (s, $1 \mathrm{H}, \mathrm{NH}-\mathrm{D}_{2} \mathrm{O}$ exchangeable), 13.60 ( $\mathrm{s}, 2 \mathrm{H}, \mathrm{NH}_{2}-\mathrm{D}_{2} \mathrm{O}$ exchangeable), ppm. MS (m/z): $331\left(\mathrm{M}^{+}\right), 333(\mathrm{M}+2), 317\left(\mathrm{M}^{+}-\mathrm{O}\right), 302$ $\left(\mathrm{M}^{+}-\mathrm{O}_{2}\right), 208\left(\mathrm{M}^{+}-\mathrm{NH}-\right.$ pyridine $), 192\left(\mathrm{M}^{+}-\mathrm{O}_{2}-\mathrm{NH}-\right.$ pyridine$\mathrm{NH}_{2}$ ). Found, \%: C; H; N. C 49.77; H 2.96; N, 21.15. $\mathrm{C}_{14} \mathrm{H}_{10} \mathrm{ClN}_{5} \mathrm{O}_{3} \mathrm{C}, 50.69$; H, 3.04; N, 21.11.

\subsubsection{3,3-Dimethyl-1-Oxo-1,2,3,4-Tetrahydrophenazine 5,10-Dioxide 5}

A mixture of 5(6)-substituted benzofuroxane 1a $(0.01$ mol) and dimidone $(0.01 \mathrm{~mol})$ in ethanol $(50 \mathrm{~mL})$ was stirred and heated at $60^{\circ} \mathrm{C}$, while ammonia gas was bubbled into the solution for $1 \mathrm{hr}$. The solution was stirred at room temperature for another $11 \mathrm{hr}$. The solvent was then removed and finally a yellow precipitate was obtained which was recrystallized out from ethanol.

Yield $75 \%$; mp. $267^{\circ} \mathrm{C}-268^{\circ} \mathrm{C}$. IR $\left(\mathrm{KBr}, \mathrm{cm}^{-1}\right): 1670$ $(\mathrm{C}=\mathrm{O}), 1627(\mathrm{C}=\mathrm{N}), 1319,1340(\mathrm{NO}) .{ }^{1} \mathrm{H}$ NMR spectrum (DMSO-d6), $\delta$, ppm 1.16, $1.22\left(2 \mathrm{~s}, 6 \mathrm{H}, 2 \mathrm{CH}_{3}\right), 3.05$ $\left(\mathrm{s}, 2 \mathrm{H}, \mathrm{H}_{2}\right), 3.34\left(\mathrm{~s}, 2 \mathrm{H}, \mathrm{H}_{4}\right), 7.76\left(\mathrm{~m}, 2 \mathrm{H}, \mathrm{H}_{7}\right), 7.86(\mathrm{~m}$, $\left.2 \mathrm{H}, \mathrm{H}_{8}\right), 8.43\left(\mathrm{~d}, 1 \mathrm{H}, \mathrm{H}_{6}\right), 8.61\left(\mathrm{~d}, 1 \mathrm{H}, \mathrm{H}_{9}\right)$. Found, \%: C; H; N. C 65.17; H 5.36; N, 11.12. $\mathrm{C}_{14} \mathrm{H}_{14} \mathrm{~N}_{2} \mathrm{O}_{3}, \mathrm{C}, 65.11$; H, 5.46; N, 10.85 .

\subsubsection{1-Oxo-11H-indeno[1,2-b]Quinoxaline 5,10-Dioxide 6}

A solution of 5(6)-substituted benzofuroxane 1a and indanedione $(0.01 \mathrm{~mol})$ inethanol $(50 \mathrm{Ml})$ was stirred for $12 \mathrm{hr}$ at room temperature in the presence of morpholine as a catalyst. The red precipitate separated after standing at room temperature for an additional $12 \mathrm{hr}$. The product was finally filtered and recrystallized out from ethanol.

Yield $61 \%$; mp. $222^{\circ} \mathrm{C}-225^{\circ} \mathrm{C}$. IR $\left(\mathrm{KBr}, \mathrm{cm}^{-1}\right): 1715$
$(\mathrm{C}=\mathrm{O}), 1642(\mathrm{C}=\mathrm{N}), 1321,1345(\mathrm{NO}) .{ }^{1} \mathrm{H}$ NMR spectrum (DMSO-d6), $\delta$, ppm 7.68 (m, 2H, $\mathrm{H}_{7}+\mathrm{H}_{8}$ ), 7.73 (d, 2H, $\left.\mathrm{H}_{6}+\mathrm{H}_{9}\right), 8.00\left(\mathrm{~m}, 1 \mathrm{H}, \mathrm{H}_{3}\right), 8.03\left(\mathrm{~m}, 1 \mathrm{H}, \mathrm{H}_{2}\right), 8.35(\mathrm{~d}, 2 \mathrm{H}$, $\left.\mathrm{H}_{4}\right), 8.85\left(\mathrm{~d}, 1 \mathrm{H}, \mathrm{H}_{1}\right), \mathrm{MS}(\mathrm{m} / \mathrm{z}): 264\left(\mathrm{M}^{+}\right), 248\left(\mathrm{M}^{+}-\mathrm{O}\right)$, $232\left(\mathrm{M}^{+}-\mathrm{O}_{2}\right), 204\left(\mathrm{M}^{+}-\mathrm{CO}\right)$. Found, \%: C; H; N. C 69.01; H 2.66; N, 11.12. $\mathrm{C}_{15} \mathrm{H}_{8} \mathrm{~N}_{2} \mathrm{O}_{3} \mathrm{C}, 68.18 ; \mathrm{H}, 3.05 ; \mathrm{N}, 10.60$.

\subsection{Antimicrobial and Antifungal Assays}

Antimicrobial activity was determined using the agar well diffusion assay method as described by [20]. The tested organisms were subcultured on nutrient agar medium (Oxoid laboratories, UK) for bacteria and Saboroud dextrose agar (Oxoid laboratories, UK) for fungi. Ampicillin and Gentamycin were used as a positive control for bacterial strains. Amphotericin B was used as a positive control for fungi. The plates were done in triplicate. Bacterial cultures were incubated at $37^{\circ} \mathrm{C}$ for $24 \mathrm{~h}$ while the other fungal cultures were incubated at $\left(25^{\circ} \mathrm{C}-30^{\circ} \mathrm{C}\right)$ for 3 - 7 days. Antimicrobial activity was determined by measurement zone of inhibition [21].

\section{Determination of MIC}

The minimum inhibitory concentration (MIC) of the samples was estimated for each of the tested organisms in triplicates. Varying concentrations of the samples (1000 - $0.007 \mu \mathrm{g} / \mathrm{ml})$, nutrient broth were added and then a loopful of the test organism previously diluted to 0.5 McFarland turbidity standard was introduced to the tubes. A tube containing broth media only was seeded with the test organisms to serve as control. Tubes containing tested organisms cultures were then incubated at $37^{\circ} \mathrm{C}$ for $24 \mathrm{~h}$ while the other fungal cultures were incubated at $\left(25^{\circ} \mathrm{C}-30^{\circ} \mathrm{C}\right)$ for $3-7$ days. The tubes were then examined for growth by observing for turbidity [22].

\section{REFERENCES}

[1] Y. Deepika, P. Surendra, N. K. Sachin and S. Shewta, "Biological Activity of Quinoxaline Derivatives," International Journal of Current Pharmaceutical Review and Research, Vol. 1, No. 3, 2011, pp. 33-46.

[2] B. Ganley, G. Chowdhury, J. Bhansali, J. S. Daniels and K. S. Gates, "Redox-Activated, Hypoxia-Selective DNA Cleavage by Quinoxaline 1,4-di-N-oxide," Bioorganic and Medicinal Chemistry, Vol. 9, No. 9, 2001, pp. 23952401. http://dx.doi.org/10.1016/S0968-0896(01)00163-8

[3] R. G. Glushkov, T. I. Vozyakova, E. V. Adamskaya, S. A. Aleinikova, T. P. Radkevich, L. D. Shepilova, E. N. Padeiskaya and T. A. Gus'kova, "Synthesis and Antibacterial Activity of New Derivatives of 1,4-di-N-Oxides of Quinoxaline," Pharmaceutical Chemistry Journal, Vol. 28, No. 1, 1994, pp. 17-20. http://www.springer.com/10.1007/BF02218945

[4] E. Vicente, R.Villar, A. Burguete, B. Solano, S. PérezSilanes, I. Aldana, J. A. Maddry, A. J. Lenaerts, S. G. 
Franzblau, S. Cho, A. Monge and R. C. Goldman, "Efficacy of Quinoxaline-2-Carboxylate 1,4-Di- $N$-Oxide Derivatives in Experimental Tuberculosis," Antimicrobial Agents and Chemotherapy, Vol. 52, No. 9, 2008, pp. 3321-3326. http://dx.doi.org/10.1128/AAC.00379-08

[5] M. Loriga, A. Nuvole, G. Paglietti, G. Fadda and S. Zanetti, "2-Phenyl-6(7)-R Substituted Quinoxalines N-Oxides. Synthesis, Structure Elucidation and Antimicrobial Activity," European Journal of Medicinal Chemistry, Vol. 25, No. 6, 1990, pp. 527-532. http://dx.doi.org/10.1016/0223-5234(90)90147-U

[6] D. Benitez, M. Cabrera, P. Hernández, L. Boiani, M. L. Lavaggi, R. Di Maio, G. Yaluff, E. Serna, S. Torres, M. E. Ferreira, N. Vera de Bilbao, E. Torres, S. Pérez-Silanes, B. Solano, E. Moreno, I. Aldana, A. López de Ceráin, H. Cerecetto, M. González and A. Monge, "Trifluoromethylquinoxaline N,N'-dioxides as Anti-Trypanosomatid Agents. Identification of Optimal Anti-T. Cruzi Agents and Mechanism of Action Studies," Journal of Medicinal Chemistry, Vol. 54, No. 10, 2011, pp. 3624-3636. http://dx.doi.org/10.1021/jm2002469

[7] G. Aguirre, H. Cerecetto, R. Di Maio, M. González, M. E. Alfaro, A. Jaso, B. Zarranz, M. A. Ortega, I. Aldana and A. Monge-Vega, "Quinoxaline N,N'-Dioxide Derivatives and Related Compounds as Growth Inhibitors of Trypanosoma Cruzi. Structure-Activity Relationships" Bioorganic and Medicinal Chemistry Letters, Vol. 14, No. 14, 2004, pp. 3835-3839.

http://dx.doi.org/10.1016/j.bmcl.2004.04.088

[8] A. Carta, M. Loriga, G. Paglietti, A. Mattana, P. L. Fiori, P. Mollicotti, L. Sechi and S. Zanetti, "Synthesis AntiMycobacterial, Anti-Trichomonas and Anti-Candida in Vitro Activities of 2-Substituted-6,7-Diflouro-3-Methylquinoxaline 1,4-dioxides," European Journal of Medicinal Chemistry, Vol. 39, No. 2, 2004, pp. 195-203. http://dx.doi.org/10.1016/j.ejmech.2003.11.008

[9] S. Ancizu, E. Moreno, E. Torres, A. Burguete, S. PérezSilanes, D. Benítez, R. Villar, B. Solano, A. Marín, I. Aldana, H. Cerecetto, M. González and A. Monge, "Heterocyclic-2-Carboxylic Acid (3-Cyano-1,4-di-Noxidequinoxalin-2-yl) Amide Derivatives as Hits for the Development of Neglected Disease Drugs," Molecules, Vol. 14, No. 6, 2009, pp. 2256-2272.

[10] A. Jaso, B. Zarranz, I. Aldana and A. Monge, "Synthesis of New Quinoxaline-2-Carboxylate 1,4-Dioxide Derivatives as Anti-Mycobacterium Tuberculosis Agents," Journal of Medicinal Chemistry, Vol. 48, No. 6, 2005, pp. 2019-2025. http://dx.doi.org/10.1021/jm049952w

[11] E. Moreno, S. Ancizu, S. Pérez-Silanes, E. Torres, I. Aldana and A. Monge, "Synthesis and Antimycobacterial Activity of New Quinoxaline-2-Carboxamide 1,4-di-NOxide Derivatives," European Journal of Medicinal Chemistry, Vol. 45, No. 10, 2010, pp. 4418-4426. http://dx.doi.org/10.1016/j.ejmech.2010.06.036

[12] E. Vicente, S. Pérez-Silanes, L. M. Lima, S. Ancizu, A. Burguete, B. Solano, R. Villar, I. Aldana and A. Monge,
"Selective Activity against Mycobacterium tuberculosis of New Quinoxaline 1,4-di-N-Oxides," Bioorganic and Medicinal Chemistry, Vol. 17, No. 2, 2009, pp. 385-389. http://dx.doi.org/10.1016/j.bmc.2008.10.086

[13] B. Zarranz, A. Jaso, I. Aldana and A. Monge, "Synthesis and Antimycobacterial Activity of New Quinoxaline-2Carboxamide 1,4-di-N-Oxide Derivatives," Bioorganic and Medicinal Chemistry, Vol. 11, No. 10, 2003, pp. 2149-2156.

http://dx.doi.org/10.1016/S0968-0896(03)00119-6

[14] A. Monge, J. A. Palop, M. Gonzaliz, F. J. Martinez Crespo, A. Lopez de Cerain, Y. Sainz and S. Narro, "New Hypoxia-Selective Cytotoxines Derived from Quinoxaline 1,4-Dioxides," Journal of Heterocyclic Chemistry, Vol. 32, No. 4, 1995, pp. 1213-1217. http://dx.doi.org/10.1002/jhet.5570320420

[15] M. J. Haddadin, G. A. gopian, C. H. Issidorides, "Synthesis and Photolysis of Some Substituted Quinoxaline di-NOxides," Journal of Organic Chemistry, Vol. 36, No. 4, 1971, pp. 514-517.

http://dx.doi.org/10.1021/jo00803a005

[16] M. A. Ortega, Y. Sainz, M. E. Montoya, A. Jaso, B. Zarranz, I. Aldana and A. Monge, "Anti-Mycobacterium tuberculosis Agents Derived from Quinoxaline-2-Carbonitrile and Quinoxaline-2-Carbonitrile 1,4-di-N-oxide," Arzneimittelforschung, Vol. 52, 2002, pp. 113-119.

[17] K. M. Amin, M. M. F. Ismail, E. Noaman, D. H. Soliman and Y. A. Ammar, "New Quinoxaline 1,4-di-N-Oxides. Part 1: Hypoxia-Selective Cytotoxins and Anticancer Agents Derived from Quinoxaline 1,4-di-N-Oxides," Bioorganic and Medicinal Chemistry, Vol. 14, 2006, pp. 6917-6923.

http://dx.doi.org/10.1016/j.bmc.2006.06.038

[18] G. W. H. Cheeseman, "Condensed Pyrazines," Wiley and Sons, New York, 1979, p. 35.

[19] B. Zarranz, A. Jaso, I. Aldana and A. Monge, "Synthesis and Anticancer Activity Evaluation of New 2-Alkylcarbonyl and 2-Benzoyl-3-Trifluoromethyl-Quinoxaline 1,4di-N-oxide Derivatives," Bioorganic and Medicinal Chemistry, Vol. 12, No. 37, 2004, pp. 3711-3721. http://dx.doi.org/10.1016/i.bmc.2004.04.013

[20] I. A. Holder and S. T. Boyce, “Agar Well Diffusion Assay Testing of Bacterial Susceptibility to Various Antimicrobials in Concentrations Non-Toxic for Human Cells in Culture," Burns, Vol. 20, 1994, pp. 426-429. http://dx.doi.org/10.1016/0305-4179(94)90035-3

[21] H. Agwa, M. M. Aly and R. Bonaly, "Isolation and Characterization of Two Streptomyces Species Produced Non Polyenic Antifungal Agents," Journal of Union Arab Bioogyl, Vol. 7, 2000, pp. 62-82.

[22] J. H. Doughari, "Antimicrobial Activity of Tamarindus indica Linn. Trop," Journal of Pharmaceutical Research, Vol. 5, No. 2, 2006, pp. 597-603. 